\title{
Encoding Immunological Memory in the Initiation of B-Cell Receptor Signaling
}

\author{
Susan K. Pierce ${ }^{1}$ and Wanli LiU ${ }^{2,3}$ \\ ${ }^{1}$ Laboratory of Immunogenetics, National Institute of Allergy and Infectious Diseases, \\ National Institutes of Health, Rockville, Maryland 20852 \\ ${ }^{2}$ MOE Key Laboratory of Protein Science, School of Life Sciences, Tsinghua University, \\ Beijing 100084, China \\ ${ }^{3}$ Collaborative Innovation Center for Diagnosis and Treatment of Infectious Diseases, The First \\ Affiliated Hospital, School of Medicine, Zhejiang University, Hangzhou 310003, China \\ Correspondence: spierce@nih.gov
}

\begin{abstract}
In one of the earliest events in the initiation of antigen-driven antibody responses, naïve, IgM-, and IgD-expressing B cells enter germinal centers where they irreversibly isotype switch to the expression of predominately IgG B-cell receptors (BCRs). The IgG-expressing B cells then undergo rounds of antigen-driven selection, ultimately exiting germinal centers as IgGexpressing memory B cells or plasma blast. This early switch from IgM to IgG begs the question: Of what advantage to the memory response is the B cell's expression of an IgG BCR? Despite convincing evidence that the expression of IgG BCRs is essential for antibody memory responses in vivo, the molecular basis of this requirement is only incompletely understood. Here we describe intrinsic features of IgG BCRs that endow memory B cells with the ability to rapidly and efficiently initiate signaling. Remarkably, efficient signaling is mediated through the cytoplasmic tail of the membrane IgG that binds to synapse associated protein 97 , a member of a large family of proteins that are best studied for their role in regulating receptor signaling in neuronal synapses. These findings underscore an interesting parallel in the mechanisms at play in encoding immunological memory and memory in the nervous system.
\end{abstract}

B-cell memory, a hallmark of adaptive immunity, is characterized by the rapid production of large amounts of high-affinity antibody $(\mathrm{Ab})$ in response to the reexposure to an antigen $(\mathrm{Ag})$. $\mathrm{Ab}$ memory is encoded in part by memory B cells (MBCs) that express BCRs containing high-affinity, isotype-switched, somatically hypermutated membrane IgG (mIgG) in contrast to naïve B cells that express exclusively mIgM-, and mIgD-containing BCRs (McHeyzer-Williams and McHeyzer-Williams 2005). In addition to the $\mathrm{mIg}$ all $\mathrm{BCRs}$ contain a heterodimer, $\operatorname{Ig} \alpha-\operatorname{Ig} \beta$ that connects BCRs to the B cell's intracellular signaling apparatus through immune-receptor tyrosinebased activation motifs (ITAMs) in their cytoplasmic tails (Reth 1992). Upon Ag binding the BCR's ITAMs become phosphorylated by Src-family kinases, particularly Lyn, leading to the binding, phosphorylation, and activation of the kinase Syk, ultimately triggering a variety of downstream signaling cascades (Cambier et al. 2007). Since $\operatorname{IgG}$ and $\operatorname{IgM}$ BCRs contain identical $\operatorname{Ig} \alpha-\operatorname{Ig} \beta$ heterodimers and differ only in their $\mathrm{mIg}$, any functional differences in the IgG versus IgM BCRs must be encoded in the $\mathrm{mIgG}$ and $\mathrm{mIgM}$ themselves. At present there is little evidence that differences in the ectodomains or transmembrane domains of $\mathrm{mIgM}$ and $\mathrm{mIgG}$ contribute to memory responses, although such a possibility cannot be ruled out. In contrast, several studies have showed a necessary role for the cytoplasmic tail of $\mathrm{mIgG}$ in $\mathrm{Ab}$ memory responses in vivo. Both $\mathrm{mIgM}$ and $\mathrm{mIgD}$ have short cytoplasmic tails, estimated to be approximately three amino acids in length. In contrast, all mIgG subclasses contain highly conserved 28 amino acid tails. The short tails of $\mathrm{mIgM}$ and $\mathrm{mIgD}$ have not been implicated in BCR signaling whereas the $\mathrm{mIgG}$ cytoplasmic domains have been shown to be both necessary and sufficient for hightitered IgG memory responses in vivo. Mice that carry mIgG1 with a truncated tail generate poor Ag-specific memory Ab responses (Kaisho et al. 1997) and similarly in adoptive transfer experiments, B cells expressing $\mathrm{mIgM}$ with IgG1 tails showed increased number of plasma blast, that enhance the magnitude of the memory $\mathrm{Ab}$ response (Martin and Goodnow 2002). Differences in the signaling functions of IgG BCRs and IgM BCRs have also been showed in vitro including enhanced calcium responses in IgG BCR expressing B cells (Wakabayashi et al. 2002; Horikawa et al. 2007; Waisman et al. 2007) and distinct gene expression profiles (Horikawa et al. 2007). Engels et al. (2009) provided a molecular basis for downstream signaling differences in IgM and IgG BCRs showing that conserved Ig tail tyrosine (ITT) motif in the $\mathrm{mIgG}$ cytoplasmic domain was phosphorylated upon BCR cross-linking and recruited the adaptor, Grb2, to the IgG $\mathrm{BCR}$ resulting in enhanced calcium responses and B-cell proliferation.

Collectively these studies provide convincing evidence that the IgG tail plays a central role in memory responses in vivo and in downstream signaling in B cells in vitro. In 
recent years, primarily through the application of highresolution live cell imaging technologies, we have learned a great deal about the earliest events in the initiation of BCR signaling that occur within seconds of the binding of $\mathrm{Ag}$ to the BCR and lead to the formation of BCR microclusters and ultimately the B-cell immunological synapse (Pierce and Liu 2010). These early events depend, in part, on intrinsic features of the BCR, raising the question: Do intrinsic features of the IgG BCR influence the initiation of B-cell signaling in MBCs? Here we first discuss current views of the initiation of BCR signaling and then describe recent evidence that the cytoplasmic tail of the $\mathrm{mIgG}$ plays a central role in facilitating the initiation of BCR signaling in MBCs.

\section{CURRENT VIEWS OF THE EARLY EVENTS IN THE INITIATION OF BCR SIGNALING}

Although it is well established that the binding of Ag to the BCR triggers signaling cascades that ultimately lead to $\mathrm{B}$-cell proliferation and differentiation into $\mathrm{Ab}$ secreting plasma cells, our understanding of the fundamental process by which Ag binding to the BCR is translated into intracellular signaling remains incomplete. That the BCR responds to $\mathrm{Ag}$ binding was demonstrated in the early 1970s in seminal studies that provided dramatic images of BCRs patching and then capping in response to $\mathrm{Ag}$ (Taylor et al. 1971). In these studies and for years of subsequent studies, B cells were stimulated by Ag in solution and under these conditions B-cell responses generally required that Ags be at least bivalent if not multivalent. The atomic solution of Fabs of Ig bound to their specific Ags provided a structural explanation for the requirement for Ag multivalency (Wilson et al. 1991). Monovalent Ag binding did not induce structural changes in the Fab and thus there did not appear to be a mechanism to translate the information that $\mathrm{Ag}$ bound to the Fab to the Fc portion of the mIg and ultimately across the membrane to trigger signaling. Multivalent Ags that could physically crosslink the BCR could potentially initiate signaling by perturbing the resting state environment of the BCR and bringing the cross-linked BCRs into close molecular proximity to components of the B-cell signaling pathways. Several studies provided evidence that this was indeed the case. For example, the first kinase in the BCR signaling pathway, Lyn, a member of the Src-family kinases, is dually acetylated with saturated lipids and is associated with the plasma membrane through its acyl chains (Kovarova et al. 2001). The saturated lipids prefer the order lipid environments of what have been termed lipid rafts and associate with these. We used both biochemical techniques as well as fluorescence resonance energy transfer (FRET) in living cells to follow the association of Lyn and the BCR with lipid rafts following Ag binding. We provided evidence that in the resting state BCRs reside primarily in disordered lipid environments but upon physical cross-linking by Ags the BCRs coalesce lipid rafts around them bringing Lyn into close proximity to phosphorylate the ITAMs and trigger signaling (Sohn et al. 2008). Treanor et al. (2010) provided evidence that BCR cross-linking functions to remodel the actin cytoskeleton allowing BCRs that are confined in the resting state by actin "fences" to diffuse and encounter early BCR signaling components or alternatively to escape inhibitory interactions. Using live cell imaging, these authors showed that diffusion of the BCR in resting B cells was restricted by an ezrin-actin network and disruption of the network resulted in spontaneous BCR signaling. Subsequent studies showed that BCR-triggered reorganization of the actin cytoskeleton was required for the formation of signaling active BCR clusters (Treanor et al. 2011) and that reorganization may allow for the interaction of the BCR with CD19 to facilitate signaling (Mattila et al. 2013).

These studies raise the question: At the molecular level what is meant by the physical cross-linking of BCRs by multivalent Ag? By several experimental criteria, including FRET (Tolar et al. 2005; Sohn et al. 2008), single molecule diffusion measurements (Tolar et al. 2009a; Liu et al. 2010a,b) and recently, super resolution stochastic optical reconstruction microscopy (STORM) (Mattila et al. 2013; J Lee and SK Pierce, unpubl.), the majority of BCRs in resting cells do not appear to be in higher-order oligomers, but form large clusters in response to $\mathrm{Ag}$ (Pierce and Liu 2010). However, based on biochemical analyses of immunoprecipitated BCR (Schamel and Reth 2000) and a quantitative bifluorescence complementation assay (Yang and Reth 2010), an alternative model for the effect of multivalent Ag on BCR activation has been proposed in which BCRs on resting B cells exist as autoinhibited oligomers that multivalent Ags serve to open into signaling active monomers. This deoligomerization model was attractive as although it required multivalent Ag binding it did not require that antigenic epitopes on the $\mathrm{Ag}$ be arrayed in any particular fashion, as might be predicted if multivalent Ags were required to bring BCRs into a well-defined oligomeric structure. However, both the BCR oligomerization and deoligomerization models are similar in that in both models, multivalent Ags serve the same function, namely to physically cross-link BCRs, altering their resting state organization.

The Ag valency requirement for BCR activation is important with regard to one of the fundamental functions of the BCR, namely to discern the B cells' affinity for Ag. The affinity of bivalent BCRs for multivalent Ags can be obscured by the avidity of the interaction whereas monovalent engagement of $\mathrm{Ag}$ by the BCR would be exquisitely sensitive to the BCR's affinity for the Ag. Can monovalent Ags activate B cells under any conditions? The answer appears to be yes and that although physical cross-linking of BCRs is sufficient to induce signaling, it may not always be necessary. In particular, it appears that the context in which B cells encounter Ag may influence the valency requirement. Recently, evidence has accumulated that the relevant mode by which $\mathrm{B}$ cells encounter $\mathrm{Ag}$ in vivo may not be in solution but rather on the surfaces of antigen presenting cells (APCs) (Cyster 2010). Batista et al. (2001) first described B cells responding to Ag on the surfaces of APCs in vitro resulting in the formation of 
immunological synapses. Subsequent high-resolution imaging of B cells encountering Ag on planar lipid bilayers as surrogate APCs showed B cells spreading over the bilayers as their BCRs engaged Ag ultimately triggering a contraction to form an immune synapse (Fleire et al. 2006). Intravital imaging provided dramatic views of $B$ cells encountering Ag in lymph nodes on the surfaces of macrophages and follicular dendritic cells (Cyster 2010).

We provided evidence that the valency of the Ag is not critical to BCR activation when Ags are presented on fluid lipid bilayers, as surrogates for APC surfaces (Tolar et al. 2009a). However, the mechanisms by which monovalent and multivalent Ags initiated signaling appeared distinct. We used single molecule tracking in TIRF microscopy to study the behavior of individual BCRs in living cells as they first encountered $\mathrm{Ag}$ in fluid lipid bilayers. We observed that BCRs were immobilized following monovalent Ag binding, indicating that they had oligomerized. Oligomerization was a BCR intrinsic event that did not require a signaling active BCR but was dependent on the membrane proximal $\mathrm{C} \mu 4$ domain of the mIgM ectodomain. We hypothesized that the membrane proximal domain contained an oligomerization face that was not exposed in the resting BCR but became accessible when a force was applied to the BCR as it engaged Ag on an opposing membrane (Fig. 1). Consistent with this hypothesis, the $\mathrm{C} \mu 4$ domain when expressed on the Bcell surface with the $\operatorname{Ig} \alpha-\operatorname{Ig} \beta$ heterodimer spontaneously oligomerized and spontaneously signaled. These findings suggested a novel function for the BCR in B-cell activation in which the BCRs rather than serving to passively bind to Ag allowing BCR cross-linking, actively respond to Ag to promote their own oligomerization (Fig. 1). These findings lead us to propose a "conformation-dependent" model for BCR oligomerization (Tolar et al. 2009b; Pierce and Liu 2010; Tolar and Pierce 2010) in which in the resting state BCRs would not be competent to dimerize with other BCRs it randomly bumped. Binding of monovalent Ag on an APC surface would force a conformational change in the mIgM's $\mathrm{C} \mu 4$ domain bringing it into a dimerization-competent form such that when two Ag-engaged BCRs bumped they would dimerize. We observed that BCRs oligomerized equivalently in response to monovalent and multivalent Ags but by what appeared
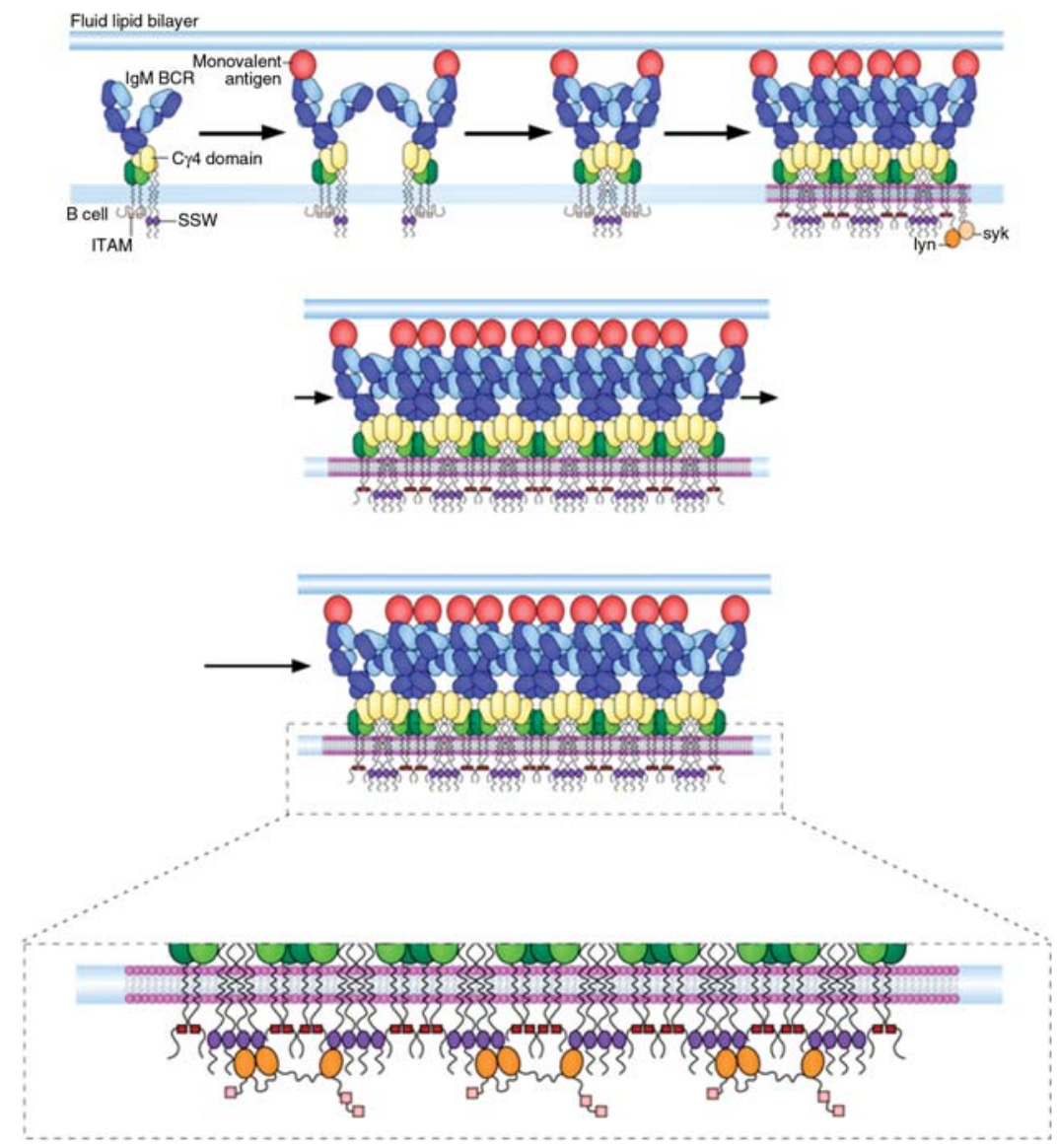

Figure 1. A view of the early events in the initiation of IgG BCR signaling. From top right: BCRs exist on the B-cell surface primarily as monomers in a dimerization incompetent form. Binding to monovalent Ag on the surface of an APC forces the BCR into a dimerization competent form such that when two Ag-engaged BCRs randomly bump they dimerize. Dimers cluster and alter the local lipid environment recruiting Lyn to phosphorylate ITAMs in the IgG BCR tails. BCR clusters continue to grow, and the resulting concentration of SSVV motif-containing $\mathrm{mIgG}$ tails facilitates binding to SAP97 through its PDZ domains that stabilize the IgG BCR clusters allowing robust signaling. 
to be distinct mechanisms. Unlike the response to monovalent $\mathrm{Ag}$ the response to multivalent $\mathrm{Ag}$ did not require the $\mathrm{C} \mu 4$ of the mIgM (Tolar et al. 2009a).

We conclude from these studies that although multivalent Ags can trigger BCR signaling, they are not necessary for BCR activation particularly in the case of B-cell recognition of Ag on APCs. Moreover, the mechanism of monovalent- versus multivalent-Ag induced signaling may not be identical. Indeed, additional recent observations also suggest that signaling induced by monovalent Ags may not be identical to that induced by multivalent Ags. Although initiation of signaling by Ag in solution generally requires that the Ag be multivalent, recent studies by Mukherjee et al. (2013) showed that monovalent hen egg lysozyme (HEL) can induce B-cell signaling. However, monovalent HEL and multivalent HEL in solution differed in their dependence on the requirement for Src-family kinases with monovalent-Ag-induced signaling requiring Src-family kinases and multivalent-Ag-induced signaling being relatively independent of Srcfamily kinases. In contrast, an earlier study by Weber et al. (2008) showed that triggering B-cell spreading responses and the initiation of BCR signaling by membrane bound multivalent Ags required Src-family kinases. Similarly, Depoil et al. (2008) showed that initiation of signaling by membrane bound Ags differed from that by Ag in solution by the requirement for the coreceptors CD19 that they showed colocalization with Ag-induced BCR microclusters.

\section{INTRINSIC FEATURES OF THE IgG BCR THAT ENHANCE INITIATION OF MBC SIGNALING}

To identify intrinsic features of $\mathrm{mIgG}$ and $\mathrm{mIgM}$ that might contribute to $\mathrm{Ab}$ memory responses we applied high-resolution live cell imaging to investigate the initiation of BCR signaling in B-cell lines expressing either $\mathrm{mIgM}$ or $\mathrm{mIgG}$ with identical VH and VL chains in response to monovalent Ag presented in fluid lipid bilayers. We observed that as compared to IgM BCRs, IgG BCRs formed more oligomers, more readily and these oligomers grew as microclusters more rapidly forming larger clusters that were more effective at recruiting the first kinases in the signaling cascade (Liu et al. 2010b). In terms of the growth of BCR clusters, comparing the accumulation of $\mathrm{Ag}$ in the clusters as compared to the accumulation of BCRs we observed that IgG BCR clusters accumulated significantly more BCR than Ag in contrast to IgM BCR clusters that accumulated equivalent amounts of BCR and Ag. This finding suggests that IgG BCRs may have an Agindependent mechanism to enhance cluster size and signaling amplitude. These early events were also dependent on the affinity of the BCR for Ag, but for BCRs with identical VH and VL, IgG BCRs were more efficient in progressing through early events in the initiation of signaling as compared to IgM BCRs (Liu et al. 2010a).

We determined that oligomerization of IgG BCRs in response to monovalent $\mathrm{Ag}$ in fluid planar lipid bilayers was dependent on the membrane proximal $\mathrm{C} \gamma 3$ domain of $\mathrm{mIgG}$, analogous to the requirement for $\mathrm{C} \mu 4$ in IgM BCR oligomerization (Tolar et al. 2009a). However, the spontaneous oligomerization of $\mathrm{C} \gamma 3$ and $\mathrm{C} \mu 4$ expressed on Bcell surfaces was similar suggesting that differences in the ectodomains of IgM and IgG BCRs may not control the efficiency of the initiation of signaling. Subsequent studies of BCRs in which the ecto-, trans-, and intracellulardomains of $\mathrm{mIgM}$ and $\mathrm{mIgG}$ were swapped or mutated indicated that the membrane proximal 15 amino acids of the cytoplasmic tail of $\mathrm{mIgG}$ was solely responsible for the efficiency of these earliest IgG BCR activation events (Liu et al. 2010b). Alignment of the known sequences of the cytoplasmic domains of mIgGs from different species shows a remarkable degree of conservation (Fig. 2). The ITT motif identified by Engels et al. (2009) that is phosphorylated upon BCR cross-linking and recruits the downstream adaptor Grb2 to the BCR is also conserved. However, sequence conservation is greatest in the membrane proximal region of $\mathrm{mIgG}$ molecules that contains a putative type I postsynaptic density 95 (PSD95)/disc large (Dlg)/zona occludens 1 (PDZ) domain-binding motif, X-S/T-X-V with SSVV as the consensus motif sequence. Interestingly, even the membrane proximal region of IgY molecules contain this putative PDZ domainbinding motif with STSV as the consensus sequence (Fig. 2). In contrast the tail of mIgE that can function in BCRs is different in this region although it contains the ITT motif (Fig. 2). This suggests that enhanced initiation of BCR signaling may be a unique feature of IgG BCRs but amplification of signaling by the ITT motif may be a shared feature of isotype-switched BCRs.

A peptide representing the membrane proximal 15 amino acids of the mIgG1 tail was used to identify binding partners in B-cell lysates. This analysis identified a PDZ domain-containing member of the conserved MAGUK family of molecular adaptors called synapse-associated protein 97 (SAP97) (Liu et al. 2012). SAP97 was of immediate interest as it is a well-studied scaffold protein in the nervous system where it has been shown to bind to the cytoplasmic tails of neuronal $N$-methyl-D-aspartic acid (NMDA) and $\alpha$-amino-3-hydroxy-5-methyl-4-isoxazolepropionic acid (AMPA) receptors and regulate their surface expression in neurological synapses (Zheng et al. 2011). SAP97 is expressed in B cells. We determined that more SAP97 was recruited to the IgG BCR immune synapse as compared to that recruited to the IgM BCR immune synapse. Using FRET TIRF microscopy we showed that SAP97 only came in to close molecular proximity to the tail of IgG BCRs and not to the IgM BCRs. Knocking down SAP97 using shRNA technology greatly diminished the ability of IgG BCRs to oligomerize and for the resulting clusters to grow and recruit kinases in the signaling pathway but had no effect on the function of IgM BCRs. Mutational studies showed that the SSVV motif in the mIgG tail was responsible for the efficient signal initiation by the IgG BCR. Studies of the kinetics of Ag-driven BCR cluster growth in wild-type and SAP97-deficient B cells showed that SAP97's function was not required early in oligomerization and clustering 


$\begin{array}{ll}\text { mIgG1 } & \text { Mouse } \\ \text { mIgG2a } & \text { Mouse } \\ \text { mIgG2b } & \text { Mouse } \\ \text { mIgG3 } & \text { Mouse } \\ \text { mIgG } & \text { Rat } \\ \text { mIgG1 } & \text { Human } \\ \text { mIgG2 } & \text { Human } \\ \text { mIgG3 } & \text { Human } \\ \text { mIgG } & \text { Orangutan } \\ \text { mIgG1 } & \text { Chimpanzee } \\ \text { mIgG1 } & \text { Mustela furo } \\ \text { mIgG1 } & \text { Vicugna pacos } \\ \text { mIgG1b } & \text { Vicugna pacos } \\ \text { mIgG2b } & \text { Vicugna pacos } \\ \text { mIgG2c } & \text { Vicugna pacos } \\ \text { mIgG } & \text { Camelus dromedarius } \\ \text { mIgG1a } & \text { Llama } \\ \text { mIgG1b } & \text { Llama } \\ \text { mIgG2b } & \text { Llama } \\ \text { mIgG2c } & \text { Llama } \\ \text { mIgG2a Came1 } \\ \text { mIgG1 } & \text { Horse } \\ \text { mIgG3 } & \text { Equus caballus } \\ \text { mIgG1 } & \text { Cow } \\ \text { mIgG1 } & \text { Dog } \\ \text { mIgG1 } & \text { Pig } \\ \text { mIgG3 } & \text { Tupaia chinensis } \\ \text { mIgG1 } & \text { Opossum } \\ \text { mIgG } & \text { Dolphin } \\ \text { mIgG1 } & \text { Heterocephalus glaber } \\ \text { mIgG2 } & \text { Heterocephalus glaber } \\ \text { mIgG1 } & \text { Cricetulus griseus } \\ \text { mIgG2a Cricetulus griseus } \\ \text { mIgG3 } & \text { Cricetulus griseus } \\ \text { mIgG3 } & \text { Bos grunniens mutus } \\ \text { mIgG1 } & \text { Platypus } \\ \text { mIgG2 } & \text { Platypus } \\ \text { mIgG1 } & \text { Chelonia mydas } \\ \end{array}$

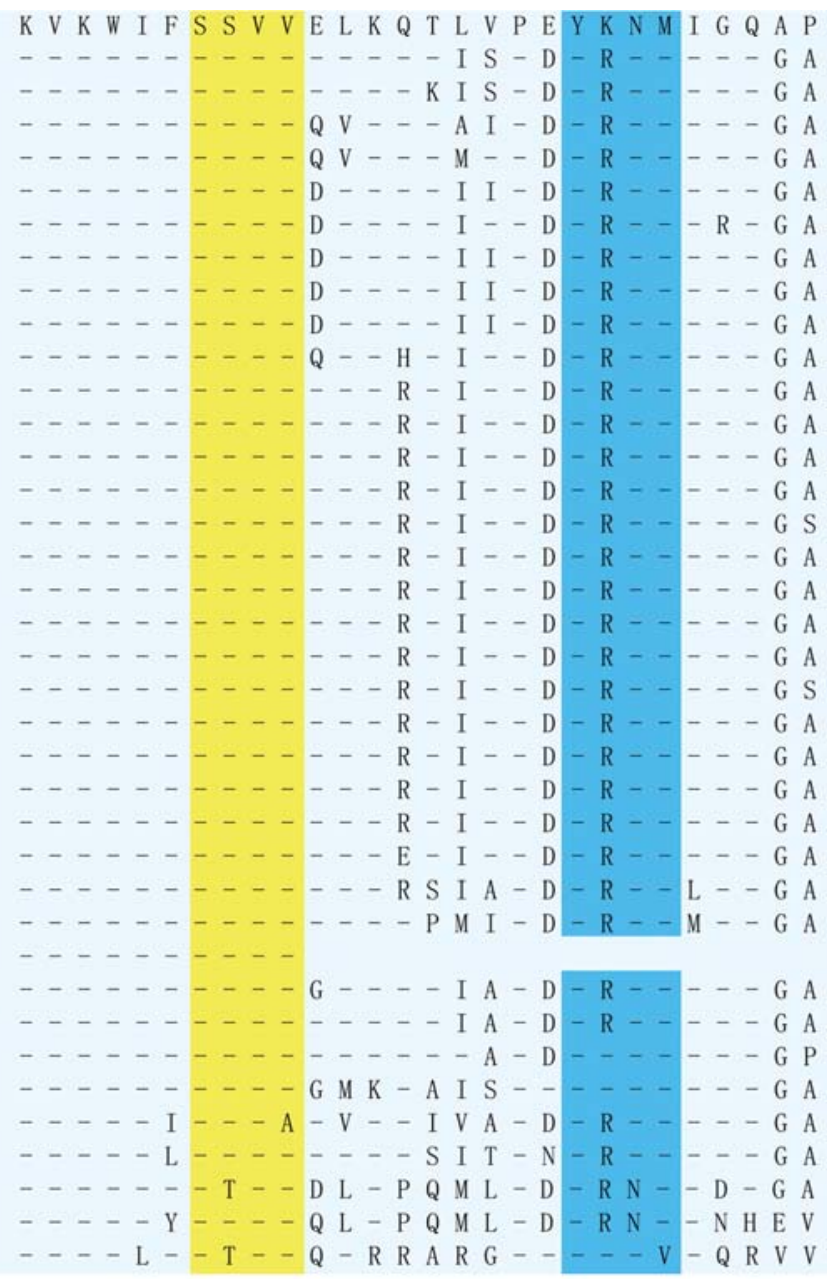

\begin{tabular}{|c|c|c|}
\hline & is s & $\cdots-L_{-P}$ T - Q - A S G S D $--\mathrm{V}-\mathrm{Q}$ R V V \\
\hline $\mathrm{mIo}$ & lus sic & $--V-K D-V$ \\
\hline IgY3 & lus sia & $-V-Q R V$ \\
\hline & sit & $-V-Q R V$ \\
\hline & & $\ldots-\cdots-M Q-\cdots R G-V$ \\
\hline 3 & sir & $\ldots-\cdots \mathrm{T}-\mathrm{V} Q-\cdots$ \\
\hline $\mathrm{IgY}$ & & 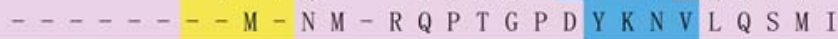 \\
\hline
\end{tabular}

\begin{tabular}{|c|c|c|}
\hline $\mathrm{mIgM}$ & Mouse & --- \\
\hline $\mathrm{mIgD}$ & Mouse & --- \\
\hline $\mathrm{mIgA}$ & Mouse & $T--R G P F G S K-V P Q Y$ \\
\hline $\mathrm{mIgE}$ & Mouse & $\cdots-V L-T P M Q D T P Q T F Q D-A-I L Q T R A$ \\
\hline
\end{tabular}

Figure 2. An alignment of the cytoplasmic tails of mIgs. Shown are alignments of the Ig tail sequences available in NCBI. The SSVV motifs are highlighted in yellow and the ITT motifs in blue.

of the BCR (before $5 \mathrm{~min}$ ) but became essential for efficient clustering subsequently.

These results lead us to propose that the efficiency with which IgG BCRs initiate signaling is encoded in its highly conserved cytoplasmic tail through its interaction with SAP97 (Fig. 1). It will be of considerable interest to determine how SAP97 functions in IgG BCR signaling. In addition to its ability to bind to the tail of $\mathrm{mIgG}$ that may serve to stabilize IgG BCR clusters at the immune synapse, SAP97 also binds to key kinases in the BCR signaling pathway including p38 and PTEN and elements of the ezrin-actin cytoskeleton all of which could influence signaling. It will also be of interest to determine how SAP97 influences Ab responses in vivo. We predict that SAP97-deficient B cells will isotype switch and undergo Ag-driven affinity maturation normally. However, SAP97-deficient MBCs would not have a competitive advantage over IgM-expressing B cells in recall responses or in adoptive transfer experiments. To test this hypothesis we have generated mice in which SAP97 is conditionally knocked out in all CD $19^{+} \mathrm{B}$ cells or in germinal center B cells expressing activation-induced deaminase (AID). Re- 
cent experiments by Hawkins et al. (2013) show that fetal liver chimeric mice reconstituted with SAP97-deficient cells have normal proportions of T cells and B cells, suggesting that haematopoiesis is SAP97 independent, a finding that is largely consistent with the results of analyses of SAP 97 T-cell lineage-deficient mice. Primary and secondary IgG Ab responses to the T-cell-dependent Ag, NP$\mathrm{KLH}$, were assessed in these fetal liver chimeric mice. Both SAP97-deficient and wild-type chimeric mice appeared to produce similar levels of total and high-affinity NP-specific Abs in both the primary and secondary responses although there appeared to be a trend toward SAP97-deficient mice producing less high-affinity $\mathrm{Ab}$ in secondary responses. It will be of interest to determine if the SAP97-deficient B cells that developed from fetal liver in these chimeric mice show the impairment in early events in the initiation of signaling we described for B cells in which SAP 97 was knocked down acutely in mature cells in vitro (Liu et al. 2012).

\section{IgG BCRs IN HUMAN MBCs}

The studies described above were performed in B-cell lines or in mouse primary B cells induced in vitro to switch to IgG raising an important question: Are the behaviors of IgG and IgM BCRs observed in undifferentiated $B$ cells manifest in the cellular background of naïve $\mathrm{B}$ cells and MBCs? To address this question we studied peripheral blood IgM-expressing, $\mathrm{CD} 27^{-}$naïve $\mathrm{B}$ cells and IgG-expressing, CD27 $7^{+} \mathrm{MBCs}$ in response to Fab of light chain-specific Abs in fluid lipid bilayers (Davey and Pierce 2012). In most regards the BCRs behaved as predicted from the studies in cell lines. As compared to IgM BCRs, IgG BCRs oligomerized more efficiently, the resulting BCR clusters grew more rapidly to greater sizes and recruited greater quantities of the early signaling kinases. Moreover, SAP97 was recruited to IgG BCR immune synapse and more SAP97 colocalized with IgG BCRs following Ag engagement as compared to IgM BCRs (Liu et al. 2012). Human MBCs also showed the same interesting Ag-independent recruitment of $\mathrm{IgG}$ BCRs to clusters that may serve to amplify signaling. However, there were differences in naïve and MBCs not observed in $\mathrm{mIgM}$ and $\mathrm{mIgG}$-expressing cell lines. In resting MBCs the IgG BCRs were less mobile as compared to IgM BCRs in resting naïve B cells suggesting that IgG BCRs on the surface of MBCs were either oligomerized or confined in some way by association with other proteins or the actin cytoskeleton. It is clear that this behavior is not unique to IgG BCRs as IgD BCRs have been reported to be less mobile than IgM BCRs (Treanor et al. 2010). The IgG BCRs in resting MBCs were also associated with significantly more activated PI3K as compared to IgM BCRs in resting naïve B cells. PI3K plays a central role in tonic BCR signaling (Srinivasan et al. 2009) suggesting that IgG BCRs in MBCs may be more signaling active. Together these observations suggested that the spatial organization of BCRs or of components of the signaling pathway were different on naïve B cells and MBCs, possibly to facilitate signaling in MBCs.

To understand the spatial organization of IgG BCRs on MBCs as compared to IgM BCRs on naïve B cells, we have recently initiated studies using STORM super resolution microscopy. Using STORM Mattila et al. (2013) provided evidence that in resting B cells IgD BCRs were more likely to be in nanoclusters on the cell surface as compared to IgM BCRs. Our initial studies indicate that both IgM and IgG BCRs exist primarily as monomers in resting cells with a small subpopulation of dimers that do not appear to be arrayed differently on cell surfaces. Ag engagement resulted in both IgM and IgG BCR clusters but interestingly, the predominant form was dimers in both cases. This suggests that what appears as large aggregates of BCRs on the B-cell surface following Ag binding at low resolution are in fact dimers confined to an area of the B-cell surface. If so, this would have important implications for the molecular nature of the basic BCR signaling unit. Future use of super resolution microscopy should reveal how BCRs are arrayed on the surface of naïve B cells and MBCs in relationship to inhibitory and activating coreceptors. Such knowledge may contribute to a better understanding of how to control BCR function in autoimmune disease and in B-cell cancers.

\section{REFERENCES}

Batista FD, Iber D, Neuberger MS. 2001. B cells acquire antigen from target cells after synapse formation. Nature 411: 489-494. Cambier JC, Gauld SB, Merrell KT, Vilen BJ. 2007. B-cell anergy: From transgenic models to naturally occurring anergic B cells? Nat Rev Immunol 7: 633-643.

Cyster JG. 2010. B cell follicles and antigen encounters of the third kind. Nat Immunol 11: 989-996.

Davey AM, Pierce SK. 2012. Intrinsic differences in the initiation of B cell receptor signaling favor responses of human $\mathrm{IgG}^{+}$memory B cells over IgM ${ }^{+}$naive B cells. J Immunol 188: $3332-3341$.

Depoil D, Fleire S, Treanor BL, Weber M, Harwood NE, Marchbank KL, Tybulewicz VL, Batista FD. 2008. CD19 is essential for $\mathrm{B}$ cell activation by promoting $\mathrm{B}$ cell receptor-antigen microcluster formation in response to membrane-bound ligand. Nat Immunol 9: 63-72.

Engels N, Konig LM, Heemann C, Lutz J, Tsubata T, Griep S, Schrader V, Wienands J. 2009. Recruitment of the cytoplasmic adaptor Grb2 to surface $\operatorname{IgG}$ and $\mathrm{IgE}$ provides antigen receptor-intrinsic costimulation to class-switched B cells. Nat Immunol 10: 1018-1025.

Fleire SJ, Goldman JP, Carrasco YR, Weber M, Bray D, Batista FD. 2006. B cell ligand discrimination through a spreading and contraction response. Science 312: 738-741.

Hawkins ED, Oliaro J, Kallies A, Belz GT, Filby A, Hogan T, Haynes N, Ramsbottom KM, Van Ham V, Kinwell T, et al. 2013. Regulation of asymmetric cell division and polarity by Scribble is not required for humoral immunity. Nat Commun 4: 1801.

Horikawa K, Martin SW, Pogue SL, Silver K, Peng K, Takatsu K, Goodnow CC. 2007. Enhancement and suppression of signaling by the conserved tail of IgG memory-type B cell antigen receptors. J Exp Med 204: 759-769.

Kaisho T, Schwenk F, Rajewsky K. 1997. The roles of $\gamma 1$ heavy chain membrane expression and cytoplasmic tail in IgG1 responses. Science 276: 412-415. 
Kovarova M, Tolar P, Arudchandran R, Draberova L, Rivera J, Draber P. 2001. Structure-function analysis of Lyn kinase association with lipid rafts and initiation of early signaling events after Fc $\epsilon$ receptor I aggregation. Mol Cell Biol 21: 8318-8328.

Liu W, Meckel T, Tolar P, Sohn HW, Pierce SK. 2010a. Antigen affinity discrimination is an intrinsic function of the B cell receptor. J Exp Med 207: 1095-1111.

Liu W, Meckel T, Tolar P, Sohn HW, Pierce SK. 2010b. Intrinsic properties of immunoglobulin IgG1 isotype-switched B cell receptors promote microclustering and the initiation of signaling. Immunity 32: 778-789.

Liu W, Chen E, Zhao XW, Wan ZP, Gao YR, Davey A, Huang E, Zhang L, Crocetti J, Sandoval G, et al. 2012. The scaffolding protein synapse-associated protein 97 is required for enhanced signaling through isotype-switched IgG memory B cell receptors. Sci Signal 5: ra54.

Martin SW, Goodnow CC. 2002. Burst-enhancing role of the IgG membrane tail as a molecular determinant of memory. Nat Immunol 3: 182-188.

Mattila PK, Feest C, Depoil D, Treanor B, Montaner B, Otipoby KL, Carter R, Justement LB, Bruckbauer A, Batista FD. 2013. The actin and tetraspanin networks organize receptor nanoclusters to regulate B cell receptor-mediated signaling. Immunity 38: $461-474$.

McHeyzer-Williams LJ, McHeyzer-Williams MG. 2005. Antigen-specific memory B cell development. Annu Rev Immunol 23: $487-513$.

Mukherjee S, Zhu J, Zikherman J, Parameswaran R, Kadlecek TA, Wang Q, Au-Yeung B, Ploegh H, Kuriyan J, Das J, et al. 2013. Monovalent and multivalent ligation of the B cell receptor exhibit differential dependence upon Syk and Src family kinases. Sci Signal 6: ra1.

Pierce S, Liu W. 2010. The tipping points in the initiation of B cell signalling: How small changes make big differences. Nat Rev Immunol 10: 767-777.

Reth M. 1992. Antigen receptors on B lymphocytes. Annu Rev Immunol 10: 97-121.

Schamel WW, Reth M. 2000. Monomeric and oligomeric complexes of the B cell antigen receptor. Immunity 13: 5-14.

Sohn HW, Tolar P, Pierce SK. 2008. Membrane heterogeneities in the formation of $\mathrm{B}$ cell receptor-Lyn kinase microclusters and the immune synapse. $J$ Cell Biol 182: 367-379.

Srinivasan L, Sasaki Y, Calado DP, Zhang B, Paik JH, DePinho RA, Kutok JL, Kearney JF, Otipoby KL, Rajewsky K. 2009. PI3 kinase signals BCR-dependent mature B cell survival. Cell 139: 573-586.

Taylor RB, Duffus WP, Raff MC, de Petris S. 1971. Redistribution and pinocytosis of lymphocyte surface immunoglobulin molecules induced by anti-immunoglobulin antibody. Nat New Biol 233: 225-229.

Tolar P, Pierce SK. 2010. A conformation-induced oligomerization model for B cell receptor microclustering and signaling. Curr Top Microbiol Immunol 340: 155-169.

Tolar P, Sohn HW, Pierce SK. 2005. The initiation of antigeninduced B cell antigen receptor signaling viewed in living cells by fluorescence resonance energy transfer. Nat Immunol 6: $1168-1176$.

Tolar P, Hanna J, Krueger PD, Pierce SK. 2009a. The constant region of the membrane immunoglobulin mediates B cellreceptor clustering and signaling in response to membrane antigens. Immunity 30: 44-55.

Tolar P, Sohn HW, Liu W, Pierce SK. 2009b. The molecular assembly and organization of signaling active B cell receptor oligomers. Immunol Rev 232: 34-41.

Treanor B, Depoil D, Gonzalez-Granja A, Barral P, Weber M, Dushek O, Bruckbauer A, Batista FD. 2010. The membrane skeleton controls diffusion dynamics and signaling through the B cell receptor. Immunity 32: 187-199.

Treanor B, Depoil D, Bruckbauer A, Batista FD. 2011. Dynamic cortical actin remodeling by ERM proteins controls BCR microcluster organization and integrity. $J$ Exp Med 208: 1055-1068.

Waisman A, Kraus M, Seagal J, Ghosh S, Melamed D, Song J, Sasaki Y, Classen S, Lutz C, Brombacher F, et al. 2007. IgG1 $\mathrm{B}$ cell receptor signaling is inhibited by $\mathrm{CD} 22$ and promotes the development of B cells whose survival is less dependent on $\operatorname{Ig} \alpha / \beta$. J Exp Med 204: 747-758.

Wakabayashi C, Adachi T, Wienands J, Tsubata T. 2002. A distinct signaling pathway used by the IgG-containing B cell antigen receptor. Science 298: 2392-2395.

Weber M, Treanor B, Depoil D, Shinohara H, Harwood NE, Hikida M, Kurosaki T, Batista FD. 2008. Phospholipase C$\gamma 2$ and Vav cooperate within signaling microclusters to propagate $\mathrm{B}$ cell spreading in response to membrane-bound antigen. $J$ Exp Med 205: 853-868.

Wilson IA, Stanfield RL, Rini JM, Arevalo JH, Schulze-Gahmen U, Fremont DH, Stura EA. 1991. Structural aspects of antibodies and antibody-antigen complexes. Ciba Found Symp 159: 13-28; discussion 28-39.

Yang J, Reth M. 2010. Oligomeric organization of the B-cell antigen receptor on resting cells. Nature 467: 465-469.

Zheng CY, Seabold GK, Horak M, Petralia RS. 2011. MAGUKs, synaptic development, and synaptic plasticity. Neuroscientist 17: $493-512$. 


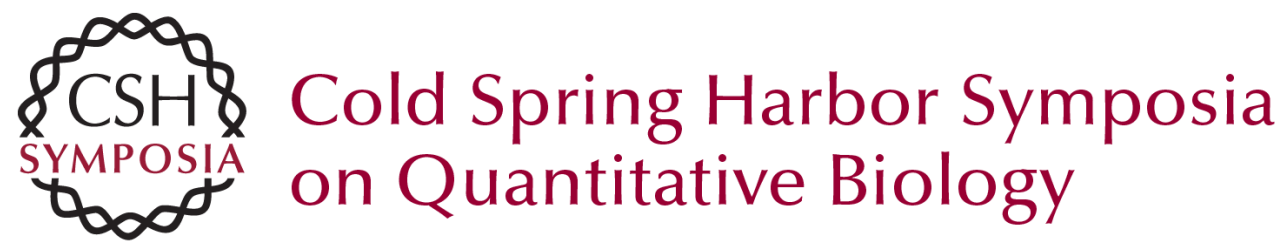

\section{Encoding Immunological Memory in the Initiation of B-Cell Receptor Signaling}

Susan K. Pierce and Wanli Liu

Cold Spring Harb Symp Quant Biol 2013 78: 231-237 originally published online October 7, 2013 Access the most recent version at doi:10.1101/sqb.2013.78.020206

References This article cites 36 articles, 13 of which can be accessed free at: http://symposium.cshlp.org/content/78/231.full.html\#ref-list-1

License

Email Alerting Receive free email alerts when new articles cite this article - sign up in Service the box at the top right corner of the article or click here. 